\title{
Die Europäische Union als außenpolitischer Akteur? Zur Kohärenz der EU-Mittelmeerpolitik und zur Rolle der Kommission
}

\author{
Peter Schlotter*
}

Die Geschichte der europäisch-nordafrikanischen Beziehungen war seit den Römischen Verträgen bis weit in die 1980er Jahre hinein eine Abfolge hehrer Versprechungen und dürftiger Ergebnisse. Erst Mitte der 1990er Jahre wagte die Europäische Union mit der ,EuroMediterranen Partnerschaft' (EMP) einen neuen Anlauf. Idee und Konzeption dieses ,Barcelona-Prozesses“ werden in der Literatur durchweg positiv bewertet: Die Komplexität des außenpolitischen Politikansatzes trage den ökonomischen und politischen Stabilitätsproblemen in der Region Rechnung und die aus dem KSZE-Prozess übernommene Korb-Struktur ermögliche einen stabilen Rahmen für die Entwicklung nachhaltiger Problemlösungsstrategien. Zudem ziele der im Barcelona-Dokument sowie in den folgenden Vereinbarungen verankerte normative Bezugspunkt ,Menschenrechte und Demokratie ' auf eine demokratische Friedenszone Europa-Mittelmeer. Und schließlich habe der partnerschaftliche ,Geist von Barcelona ' einen fairen Interessenausgleich zwischen den südlichen und nördlichen Küstenanrainern als Perspektive. ${ }^{1}$

Demgegenüber fällt das Urteil über die praktische Umsetzung der europäischen Mittelmeerpolitik meist negativ aus. Neben dem ungelösten Nahost-Konflikt und der mangelnden Kooperationsbereitschaft der Mittelmeerdrittländer (MDL) sowohl untereinander als auch gegenüber der Gemeinschaft werden dafür vor allem strukturelle Probleme auswärtiger Politikgestaltung im EU-Entscheidungssystem verantwortlich gemacht. Die komplizierten Entscheidungsvorgänge in Brüssel seien die Ursache für Einigungen auf dem kleinsten gemeinsamen Nenner und für eine Begrenzung oder sogar Lähmung der Handlungsfähigkeit der Union. ${ }^{2}$

Auch wird in der Literatur zur EG/EU-Mittelmeerpolitik überwiegend - im Kontext einer mehr oder weniger dezidiert thematisierten intergouvernementalen Sichtweise - von einer dominierenden Rolle der Mitgliedstaaten ausgegangen. Die Mittelmeerstaaten der EG/EU hätten Mitte der 1990er Jahre auf Grund ihrer spezifischen Interessenlage in Form einer

* Prof. Dr. Peter Schlotter, Hessische Stiftung Friedens- und Konfliktforschung, Frankfurt am Main, derzeit Professor für Internationale Beziehungen am Institut für Politische Wissenschaft der Universität Heidelberg.

1 Vgl. z.B. Annette Jünemann: Auswärtige Politikgestaltung im EU-Mehrebenensystem. Eine Analyse der strukturellen Probleme am Beispiel der Euro-Mediterranen Partnerschaft, in: Klaus Schubert/Gisela Müller-Brandeck-Bocquet (Hrsg.): Die Europäische Union als Akteur der Weltpolitik, Opladen 2000, S. 65-80; Klaus Schubert/Gisela Müller-Brandeck-Bocquet: Die EU und der Barcelona-Prozess - Bewertung und Perspektiven, in: integration 1/01, S. 42-57; Clara Mira Salama: The Euro-Mediterranean Partnership. Political and Economic aspects, EDPSG (European Development Policy Study Group), Discussion Paper April 2002.

2 Jörg Monar: Die interne Dimension der Mittelmeerpolitik der Europäischen Union. Institutionelle und verfahrensmäßige Probleme, in: Wulfdiether Zippel (Hrsg.): Die Mittelmeerpolitik der EU, Baden-Baden 1999, S. 65-90; Stephen Calleya/Eberhard Rhein: The Euro-Med Partnership Needs a Strong Push, in: Andreas Jacobs (Hrsg.): Euro-Mediterranean Co-operation: Enlarging and Widening the Perspective, Discussion Paper C 131, Bonn, Zentrum für Europäische Integrationsforschung 2004; Stelios Stavridis/Justin Hutchence: Mediterranean Challenges to the EU's Foreign Policy, in: European Foreign Affairs Review, 1/2000, S. 35-62; Dorothée Schmid: Optimiser le processus de Barcelone, IES (Institut d'Etudes de Sécurité) Occasional Paper 36, Paris 2002; Eric Philippart: The Euro-Mediterranean Partnership: A Critical Evaluation of an Ambitious Scheme, in: European Foreign Affairs Review, 2/2003, S. 201-220. 
neuen Mittelmeerpolitik ein Äquivalent zur Osterweiterung durchgesetzt. Sie sei damit Reflex der Interessenkonstellationen zwischen den EU-Mitgliedern gewesen. Die Kommission habe dabei eine nebensächliche Rolle gespielt. ${ }^{3}$

Diese Thesen sollen mit einer Analyse der Akteursqualität und Kohärenz der EU-Mittelmeerpolitik (unter Ausschluss der Nahostpolitik) überprüft werden. Unter einem kollektiven Akteur wird im Folgenden eine Institution verstanden, die sich durch die Wirkung gemeinsamen Handelns auszeichnet. Sie ist zwar von den Präferenzen der Mitgliedstaaten abhängig und wird von ihnen kontrolliert, verfügt aber über Kompetenzen, die von - je nach Fall unterschiedlicher - Bedeutung für die Ausführung der gemeinsamen Aktivitäten sind. ${ }^{4}$

Zur kollektiven Akteursqualität gehört kohärentes Handeln. Dieses Problem stellt sich für die Europäische Union auf eine besondere Weise, geht es doch darum - unter den Bedingungen des ,institutionellen Dualismus ' in der europäischen Außenpolitik - koordiniert und effizient eine unter den Entscheidungs- und Handlungsträgern horizontal (zwischen den EUInstitutionen und Pfeilern) und vertikal (zwischen der Union und den Mitgliedstaaten) abgestimmte und in der Durchführung koordinierte sowie inhaltlich komplexe Sachverhalte bearbeitende Außenpolitik durchzuführen. ${ }^{5}$ Viele Entscheidungen im Bereich der Mittelmeerpolitik müssen im Konsens gefällt werden, und es sind im Rahmen einer die Pfeiler übergreifenden Politik eine Reihe von EU-Institutionen daran beteiligt, die über Blockademöglichkeiten verfügen. Entscheidende Kriterien für eine kohärente Politik sind die Nutzung effizienter Verfahren sowie koordinierte, abgestimmte, inhaltlich zusammenhängende und weit gehend widerspruchsfreie Entscheidungen, die auf gemeinsamen Ordnungsvorstellungen und Leitbildern fußen und ein geschlossenes Auftreten nach außen nach sich ziehen. ${ }^{6}$ Für die Untersuchung der EU-Akteursqualität und der Kohärenz ihrer Mittelmeerpolitik sollen daher die folgenden Fragen beantwortet werden:

- Besteht prinzipielle Einigkeit der EU-Organe und ihrer Mitgliedstaaten über Strategie und Vorgehensweise der im Rahmen der EMP angestrebten Ziele?

- Ziehen die EU-Institutionen auch in der konkreten Umsetzung der EMP an einem Strang?

- Ist eine Beeinträchtigung der Kohärenz durch die Blockadehaltung beziehungsweise nationale Rückfallpositionen einiger Mitgliedstaaten zu beobachten?

- Treibt die Kommission die Mittelmeerpolitik maßgeblich und mit dem Ziel der Erhöhung der Kohärenz voran?

Konzeptionelle ,entrepreneurship‘ der Kommission bei der Vorbereitung der Euro-Mediterranen Partnerschaft

Ende der 1980er Jahre war die EG-Mittelmeerpolitik in einer tiefen Krise. Es gab keine gemeinsame Politik gegenüber den Maghrebländern, die einzelnen bilateralen Beziehungen

3 Vgl. z. B. Geoffrey Edwards/Eric Phillipart: The Euro-Mediterranean Partnership - Fragmentation and Reconstruction, in: European Foreign Affairs Review, 4/1977, S. 465-489; Federica Bicci: Actors and Factors in European Foreign Policy Making: Insights from the Mediterranean Case, EUI-Working Papers, RSC N. 2002/47, Florenz 2002; Federica Bicci: Defining European Interests in Foreign Poliy: Insights from the Mediterranean Case, ARENA Working Papers, 13/03, Oslo 2003. Demgegenüber betont Annette Jünemann, Politikgestaltung, S. 70-72, den „starken Einfluss“ der Kommission.

4 Fritz W. Scharpf: Interaktionsformen. Akteurzentrierter Institutionalismus in der Politikforschung, Opladen 2000; siehe auch Charlotte Bretherton/John Vogler: The European Union as a Global Actor, London-New York 1999.

5 Es geht im Folgenden also nicht um eine Evaluierung der Problemlösungsfähigkeit der EMP, sondern allein um die Analyse der Handlungsfähigkeit der EU unter dem Gesichtspunkt der Kohärenz und Akteursqualität.

6 Siehe hierzu neuerdings Miguel Ângel Medina Abellán: The Coherence of the European Foreign Policy: a Real Barrier of an Academic Term?, Obs-Working Paper Nr. 27, September, Barcelona (Institut Universitari d'Estudis Europeus) 2002; Pascal Gauttier: Horizontal Coherence and the External Competences of the European Union, in: European Law Journal 1/2004, S. 32-41. 
wurden gar nicht oder nur lose unter den EG-Mitgliedern abgestimmt. Insbesondere Frankreich beharrte mit seiner ,politique arabe" auf einer Sonderrolle im Mittelmeerraum. Die Aufgaben der Kommission beschränkten sich auf das Management der unterschiedlichen Agrarinteressen der EG-Mitgliedstaaten gegenüber den Mittelmeerdrittländern (MDL) und das Aushandeln von Assoziierungsabkommen, deren wirtschaftlicher Effekt von allen Beteiligten nördlich und südlich des Mittelmeers als sehr gering eingeschätzt wurde. ${ }^{7}$

In dieser Situation wurde Anfang der 1990er Jahre die Kommission aktiv. Ihre ersten Vorschläge bewegten sich noch im Rahmen der üblichen Handelspolitik und Finanzhilfe, enthielten aber auch schon einige neue Ideen: Zum einen die einer Partnerschaft, mit der die Beziehungen zu den Nicht-Mitgliedern des Mittelmeerraumes auf eine neue Stufe der gleichberechtigten Kooperation gestellt werden sollten, zum anderen die Perspektive einer Friedenszone mit den südlichen Nachbarn. Die Instabilität im südlichen und östlichen Mittelmeerraum, die durch den Golfkrieg 1991, das Aufkommen islamistischer Bewegungen und die Gewalt in Algerien nach dem Putsch der Militärs sowie durch ein sprunghaftes Bevölkerungswachstum und gewaltige wirtschaftliche und ökologische Probleme gekennzeichnet war, sollte mit einer Strategie „Freihandel + Demokratie = Entwicklung + Frieden“ stabilisiert werden. ${ }^{8}$ Außerdem schlug die Kommission vor, sie könne die Entwicklung der Zusammenarbeit (also auch in der politischen Dimension) regelmäßig überprüfen, was implizit die Konsequenz einer Kompetenzerweiterung in Politikfelder hatte, die bisher allein den Mitgliedstaaten und damit dem Rat vorbehalten waren. Die Kommission agierte dabei nicht isoliert als , policy entrepreneur ${ }^{\circ 9}$, sondern in einem politisch-gesellschaftlichen Umfeld, in dem ähnlich ausgerichtete Initiativen und Anregungen diskutiert wurden, besonders was die Politisierung der Beziehungen zu den Mittelmeer-Partnerländern anging. So forderte das Europäische Parlament eine aktive und kohärente Mittelmeerpolitik der Gemeinschaft als Teil einer gesamteuropäischen Friedenspolitik und die Einführung einer Klausel zur Demokratie und Achtung der Menschrechte in zukünftigen Verträgen.

\section{Effektives Zusammenspiel von Rat und Kommission}

Diese Konzeptionen und Strategien konnten - gemäß den vertraglichen Grundlagen - erst zur Politik der Union werden, als die Mitgliedstaaten das Thema Mittelmeer auch auf ihre Agenda gesetzt hatten. Vor allem Spanien nahm maßgeblichen Einfluss auf die Vorbereitung der neuen Europäischen Mittelmeerpolitik. ${ }^{10}$ Es konnte an die Vorschläge der Kommission sowie an Initiativen anknüpfen, die außerhalb des EG-Rahmens zwischen Spanien, Frankreich und Italien auf der einen und den Maghrebstaaten auf der anderen Seite in den

7 Vgl. zur Vorgeschichte des Barcelona-Prozesses Bichara Khader: Le partenariat euro-méditerranéen après la conférence de Barcelone, Paris 1997; Fillippos Pierros/Jacob Meunier/Stan Abrams: Bridges and Barriers. The European Union's Mediterranean Policy, 1961-1998, Aldershot 1999.

8 Vgl. Peter Schlotter: Freihandel + Demokratisierung = Entwicklung? Zur Maghrebpolitik der Europäischen Union, HSFK-Report Nr. 8/1998, Frankfurt/Main 1998.

9 Unter einem, policy entrepreneur ‘ wird ein Akteur verstanden, der mit neuen Ideen und Strategien politischen Wandel herbeiführt und ,windows of opportunity" ausnützt, um seine inhaltlichen wie auch persönlichen oder institutionellen Interessen zu verfolgen. Dies erfolgt vor allem über das , agenda setting', indem er seine Politikinitiativen in einem Netzwerk von institutionellen und außerinstitutionellen Akteuren kommuniziert und so umsetzen kann, dass sie seinen Interessen entgegenkommen. Vgl. Jonas Tallberg: The Agenda-Shaping Powers of the EU Council Presidency, in: Journal of European Public Policy, 1/2003, S. 1-19; Alexandra Krause: Die Kommission als autonomer Akteur in der GASP. Die Politik der EU gegenüber dem subsaharischen Afrika, in: Patricia Bauer/Helmut Voelzkow (Hrsg.): Die Europäische Union - Marionette oder Regisseur?, Wiesbaden 2004, S. 313-331.

10 Vgl. Richard Gillespie: Spain and the Mediterranean. Developing a European Policy towards the South, Basingstoke 2000 . 
frühen Neunzigerjahren lanciert worden waren. ${ }^{11}$ Die spanische Initiative war unter anderem deshalb erfolgreich, weil eine große gemeinsame Schnittmenge der Interessen der drei EG/ EU-Mittelmeerländer vorhanden war, die in die Verhandlungen mit den restlichen Mitgliedstaaten eingebracht werden konnten.

Auf Grund ihrer geografischen Lage waren die europäischen Mittelmeeranrainer stärker als die übrigen Mitgliedstaaten von den Entwicklungen im Süden betroffen. Weil die Ursachen der politischen Instabilität im Mittelmeerraum vor allem sozioökonomischer Natur seien, müsse - so die gemeinsame Einschätzung Spaniens, Frankreichs und Italiens - das vorrangige Ziel der Gemeinschaft sein, die großen Wohlstandsdifferenzen zwischen den Mittelmeeranrainern und den Mitgliedern der Europäischen Union zu verringern. Vor allem die französische Gesellschaft fühlte sich durch eine nicht auszuschließende Machtübernahme islamistischer Parteien in Algerien bedroht; man befürchtete, der algerische Konflikt könnte sich auf die Nachbarländer Marokko und Tunesien ausweiten und würde zu einer massenhaften Flucht nach Frankreich führen. Alle drei EU-Mittelmeerstaaten betonten, auf Grund ihrer geografischen Nähe und ihrer früheren kolonialen Beziehungen zu Nordafrika hätten sie als ,Freunde der arabischen Welt' ein besonderes Sensorium für die Probleme der Region und könnten als ,Brücke‘ Europas in den südlichen Mittelmeerraum dienen. Auch im wirtschaftlichen Bereich waren sie sich insofern einig, als sie beim Abbau protektionistischer Strukturen der Europäischen Gemeinschaft gegenüber den Mittelmeerstaaten so weit wie möglich Südfrüchte ausschließen wollten. ${ }^{12}$ Sie sahen eine zukünftige Europäische Mittelmeerpolitik als Chance, ihre Interessen in einen größeren Verbund einzubringen, weil sie glaubten, diese bilateral nicht mehr effektiv realisieren zu können. Dies galt am wenigsten für Frankreich, das noch länger an einer eigenen Rolle im Mittelmeerraum festhielt.

Für die Entscheidung für eine neue Mittelmeerpolitik musste ein Paket geschnürt werden, das außenpolitische Zielvorstellungen einer Stabilisierung des Mittelmeerraumes mit Interessen verknüpfte, die sich auf die Austarierung des Machtgefüges in der Union richteten. Nach dem Ende des Ost-West-Konflikts lag das Hauptaugenmerk der Europäischen Gemeinschaft zunächst auf der Unterstützung des Übergangs ihrer östlichen Nachbarn zu Marktwirtschaft und Demokratie. Dies sahen die EG-Mittelmeerländer als Gefahr für ihre Interessen im südlichen Mittelmeerraum an, die dadurch immer weniger (auch finanziell) berücksichtigt werden könnten. Sie versuchten daher, ihre nördlichen Partner auf die sich verschärfenden Probleme in der Mittelmeerregion aufmerksam zu machen. Hinzu kam die Angst vor einem , germanischen Europa': Dem befürchteten Machtzuwachs des vereinigten Deutschlands im Verbund mit seinen traditionellen Einflussgebieten in Ostmitteleuropa sollte als Ausgleich eine , mediterrane Perspektive ' entgegengesetzt werden. ${ }^{13}$ Alle diese Argumente wirkten auch auf die anderen Mitgliedstaaten insofern überzeugend, als diese in ihrer Mehrheit über das Schengenabkommen zu ,virtuellen Anrainern' des Mittelmeeres geworden waren. Hierdurch wie durch die Übernahme von Verantwortung im Rahmen der rotierenden Präsidentschaft und der Ratssitzungen generell wurden auch die nördlichen Staaten gezwungen, sich mit den Problemen des Mittelmeerraumes auseinander zu setzen.

11 Es handelt sich um die Vorschläge für eine „Konferenz für Sicherheit und Zusammenarbeit im Mittelmeer“ (KSZM) sowie um das 5+5-Forum, bestehend aus Spanien, Italien, Frankreich, Portugal und Malta sowie Algerien, Libyen, Tunesien, Mauretanien und Marokko.

12 Militärische Sicherheitsprobleme einer Bedrohung Europas spielten in den Überlegungen zur Lancierung einer neuen Mittelmeerpolitik nur eine geringe Rolle, sieht man von Szenarien ab, in denen eine militärisch aggressive Politik eines islamistisch-fundamentalistischen Maghreb oder Raketenangriffe aus Libyen befürchtet wurden.

13 Vgl. Esther Barbé: Paradoxes of European Foreign Policy: Balancing Europe's Eastern and Southern Dimensions, in: Jan Zielonka (Hg.), Paradoxes of European Foreign Policy, Dordrecht 1998, S. 117-130. 
Nachdem die Mitgliedstaaten bereits auf der Lissabonner Tagung des Europäischen Rates vom Juni 1992 grundsätzlich für die Vorstellungen einer neuen Mittelmeerpolitik grünes Licht gegeben hatten, erarbeitete die Kommission in diversen Mitteilungen an den Rat und das Europäische Parlament das Konzept, die Beziehungen zu Algerien, Marokko und Tunesien über die bestehende Zusammenarbeit hinaus zu einer ,euro-maghrebinischen Partnerschaft' mit dem langfristigen Ziel einer Freihandelszone weiter zu entwickeln. ${ }^{14}$ Sie basierten zunächst auf einer nüchternen Analyse der bisher mageren Ergebnisse der europäischen Mittelmeerpolitik. Kern der Partnerschaft sollte die Errichtung einer euro-mediterranen Freihandelszone sein, deren Vollendung für 2010 vorgesehen war. Nach weiteren Kommissionsvorschlägen einigten sich die Staats- und Regierungschefs im Juni 1995 in Cannes auf ein Programm, das bis in wörtliche Formulierungen hinein das wiedergab, was die Kommission vorgeschlagen hatte.

\section{Kompetenzerweiterungen für die Kommission}

Die Verhandlungen mit den südlichen und östlichen Mittelmeeranrainern über die europäischen Vorschläge waren ein Test für die in Maastricht und Amsterdam neu geschaffenen Institutionen. Seit Ende 1994 reiste die Troika in die einzelnen Länder der Region, um die Einzelheiten des EU-Angebots zu erklären. Auf der Konferenz der Außenminister der Union und der Mittelmeerpartnerländer in Barcelona am 27./28. November 1995 gelang dann die Einigung auf ein ambitioniertes Programm, das - mit Abstrichen, was die Deutlichkeit der Formulierungen bezogen vor allem auf das Erfordernis von Demokratie anging - die Positionen der Europäischen Union wiedergab. ${ }^{15}$

Ziel der ersten Säule ist die Schaffung einer politischen und sicherheitspolitischen Partnerschaft, die auf der Respektierung der Menschenrechte, auf demokratischen Normen, gesellschaftlichem Pluralismus, der Achtung der territorialen Integrität, der Methode der friedlichen Streitbeilegung, der gemeinsamen Bekämpfung des Terrorismus und der organisierten Kriminalität sowie der Förderung der Nichtverbreitung von nuklearen, chemischen und biologischen Waffen auf internationaler und regionaler Ebene beruhen soll. Kernelement der zweiten ist die schrittweise Errichtung einer Freihandelszone bis zum Jahr 2010, die durch neue bilaterale Assoziierungsabkommen zwischen der Europäischen Union und den jeweiligen Mittelmeerländern erfolgt. In der dritten Säule geht es um die Zusammenarbeit im sozialen und kulturellen Bereich. Dialog, Achtung der Kulturen und Religionen sollen gefördert, Rassismus und Fremdenfeindlichkeit bekämpft, kultureller Austausch sowie Ausbildungsund Erziehungsmöglichkeiten verbessert werden. Dieser Bereich ist politisch besonders brisant, weil hier als Zielsetzung die Förderung von Rechtsstaatlichkeit und Demokratie unter Einbeziehung der ,Zivilgesellschaften“ in den Mittelmeerländern formuliert wird. Für den Zeitraum 1995-1999 wurden 4,685 Milliarden ECU aus Haushaltsmitteln der Gemeinschaft bereitgestellt, ergänzt durch Kredite der EIB in Höhe von 3,395 Milliarden ECU und bilaterale Zuwendungen der EU-Mitgliedstaaten. 90 Prozent der EU-Haushaltsmittel waren für die Wirtschafts- und Finanzkooperation, der Rest für Programme mit gesellschaftlichen Akteuren vorgesehen. ${ }^{16}$

14 Die wichtigsten Dokumente enthält: Europäische Kommission (Hg.): Stärkung der Mittelmeerpolitik der Europäischen Union: Entwicklung einer Partnerschaft Europa-Mittelmeer, Bulletin der Europäischen Union, Beilage 2/95, Luxemburg 1995.

15 Die in diesem Aufsatz erwähnten offiziellen Dokumente zur Euro-Mediterranen Partnerschaft finden sich auf der Webseite http://www.europa.eu.int/comm/external_relations/euromed/index.htm (letzter Zugriff: 7.9.2005).

16 Für die nächste Periode (bis 2006) wurden 2000 insgesamt 5,3 Mrd. Euro zur Verfügung gestellt. 
Die Entwicklung der Europäischen Mittelmeerpolitik war die Folge einer veränderten Perzeption der Konfliktdynamik in der Mittelmeerregion sowohl durch die Kommission als auch durch die südlichen Mitgliedstaaten und einer Neuaustarierung des inneren Machtgefüges in der Union. Die Kommission handelte in enger Abstimmung mit den südlichen EGStaaten, sie stellte den Fundus von Ideen zur Verfügung, auf dem die neue Mittelmeerstrategie aufbauen konnte. Dabei nahm sie auch Ideen auf, die vom Parlament und von gesellschaftlichen Akteuren lanciert worden waren. Das Muster der Konzeptentwicklung bestand darin, auf der Grundlage einer Bestandsaufnahme aus Misserfolgen neue Konsequenzen zu ziehen. Dabei wurden keine abrupten Änderungen an der früheren Politik vorgenommen, sondern sie wurde ergänzt, verbessert und durch neue Politikdimensionen erweitert. ${ }^{17}$

Ein ,window of opportunity ‘ für diese konzeptionelle ,entrepreneurship ‘ der Kommission öffnete sich mit der neuen Interessenkonstellation unter den Mitgliedstaaten. Die Kommissionsempfehlungen wurden nicht - wie häufig üblich - vom Rat zurechtgestutzt, sondern ohne substanzielle Abstriche übernommen. Durch die Verschränkung der Politikfelder und die Politisierung der Mittelmeerpolitik (Abkehr von der reinen Handelspolitik der 1970er und 1980er Jahre) eröffnete sich der Kommission die Möglichkeit einer Kompetenzerweiterung auch auf die spezifisch außenpolitischen Dimensionen der Mittelmeerpolitik, ein Bereich, der bislang die Domäne der Mitgliedstaaten gewesen war.

Die Entscheidung für eine neue Mittelmeerpolitik fiel vertragsgemäß im Rat im Rahmen eines zwischenstaatlichen Verhandlungsprozesses. Der Rat formulierte - und dafür waren die Initiativen der Kommission richtungweisend - mit der neuen Mittelmeerpolitik den klaren Anspruch an sich selbst, als europäischer Akteur gemeinsame Zielvorstellungen zusammen mit der Kommission auf kohärente Weise umsetzen zu wollen.

\section{Die EU-Mittelmeerpolitik - Kohärenz auf der Brüsseler Ebene?}

Zentraler Bestand der Errichtung einer Freihandelszone Europa-Mittelmeer sind die - bilateral ausgehandelten - Assoziationsabkommen zwischen der Union (beziehungsweise ihren Mitgliedstaaten) und den Mittelmeerpartnern. ${ }^{18}$ Bei den Verhandlungen darüber verfolgen die einzelnen EU-Institutionen unterschiedliche Prioritäten. ${ }^{19}$ Die Kommission sieht als ihre Hauptaufgabe die Schaffung einer Freihandelszone an, die sie mit Finanzhilfen und Handelserleichterungen zur Abfederung der ökonomischen und sozialen Konsequenzen in den Partnerländern kombinieren möchte. Obwohl dies mit der Erklärung von Barcelona die prinzipielle Grundentscheidung aller Mitgliedstaaten war, kommt es bei deren Umsetzung zu internen Konflikten. So treten vor allem die südeuropäischen Staaten zwar für verstärkte Finanzhilfen, aber nur für eine begrenzte Marktöffnung ein, um ihre Landwirte vor der Konkurrenz billigerer Südfrüchte zu schützen. Die nord- und mitteleuropäischen Mitgliedstaaten, unter ihnen in erster Linie die Nettozahler, wiederum setzen sich für eine Reduktion der Finanzhilfen ein, sehen dagegen in Handelserleichterungen für Agrarprodukte für sich eher Vorteile. Die Kommission hat daher EU-intern an zwei Fronten zu kämpfen, und bei

17 Beispiele sind der Bilateralismus der Assoziationsbeziehungen, der zwar beibehalten, aber in den Kontext einer Freihandelszone gestellt wurde, die die EU und nicht nur den Maghreb, sondern den gesamten Mittelmeerraum umfassen soll, sowie die Konditionierung, ebenfalls ein Instrument, das schrittweise ausgebaut wurde.

18 Die Informationen über die europäische Mittelmeerpolitik stammen - soweit nicht auf Literatur verwiesen wird - aus Interviews mit Vertretern des Rates und der Kommission in Brüssel sowie des Auswärtigen Amtes in Berlin, die zwischen 2001 und 2004 durchgeführt wurden, sowie aus einer Auswertung der Jahrgänge 19982003 der ,Agence Europe'.

19 Dorothée Schmid: Les institutions européennes dans le fonctionnement du PEM: de la répartition des compétences à la gestion dynamique du quotidien, EuroMeSCo Paper No. 36, Lissabon 2004. 
dem EMAA-Verhandlungen versuchen die Mittelmeer-Partnerstaaten, die einzelnen Mitgliedsländer und die Kommission gegeneinander auszuspielen. Obwohl demzufolge die Interessenlagen im Bereich der wirtschaftlichen und finanziellen Partnerschaft auf Grund nationaler Vorbehalte im Detail auseinander klaffen, steht dennoch die Einrichtung der EuroMediterranen Freihandelszone generell nicht in Frage. Am Ende der Verhandlungen steht stets ein Kompromiss, der zwar nicht vollständig die Absichtserklärungen von Barcelona abbildet, aber verstärkte Finanzhilfen und zugleich Handelserleichterungen vorsieht. ${ }^{20}$

Die Kommission hat im Rahmen ihrer „Verfahrensmacht“ ${ }^{21}$ die zentrale Rolle bei der Umsetzung der Beschlüsse im Rahmen der Euro-Mediterranen Partnerschaft. ${ }^{22}$ Anfänglich war sie auf ihre neuen Aufgabe der Vorbereitung, Verwaltung und Umsetzung des BarcelonaProzesses nicht vorbereitet und hatte es versäumt, ihr Personal dafür aufzustocken. So gestand die Kommission Ende der 1990er Jahre mehrfach ein, dass es ihr nicht nur an klaren Visionen gefehlt habe, sondern auch interne Abstimmungsschwierigkeiten, Managementfehler und langwierige bürokratische Strukturen Entscheidungen verzögert oder gar verhindert hätten. Folge dieses inkohärenten Auftretens sei es gewesen, dass der Barcelona-Prozess nur eine geringe Aufmerksamkeit gefunden habe. Erst unter Romano Prodi gelang es, die Glaubwürdigkeit und Handlungsfähigkeit wieder zurückzugewinnen. Die 2000 vorgenommene Reform des MEDA-Programms, das klarer an strategischen Ziele ausgerichtet wurde als bisher, zeigte, dass die Kommission der Kritik Rechnung getragen hat und nunmehr ihre internen Prozesse effizienter gestaltet.

Im Juni 2000 beschloss der Rat - gegen hinhaltendes Desinteresse der Kommission, die einen Einflussverlust befürchtete - eine ,Gemeinsame Strategie für die Mittelmeerregion ‘. ${ }^{23}$ Vor allem die südlichen EU-Länder wollten die Bedeutung der Mittelmeerpolitik in Konkurrenz zur Osterweiterung angesichts der Debatten über die Finanzausstattung von MEDA II wieder neu hervorheben. Die Gemeinsame Strategie zielte auf eine bessere Verknüpfung der EMP mit der EU-Politik im Nahost-Konflikt, enthielt eine Bekräftigung der bisherigen europäischen Mittelmeerpolitik und betonte deren pfeilerübergreifenden Charakter, verzichtete jedoch auf jede Konkretisierung, unter anderem weil einige Mitgliedstaaten unter der Führung Großbritanniens eine Einführung des Mehrheitsprinzips in der Mittelmeerpolitik durch die Hintertür befürchteten. Sie erwies sich im Folgenden als bedeutungslos für die weitere Entwicklung des Barcelona-Prozesses.

Voraussetzung für die Umsetzung der Europäischen Mittelmeerpolitik war die Verfahrensmacht der Kommission, die sie zu einer Gestaltungsmacht erweiterte, indem sie - im Rahmen der durch die Entscheidungsmacht des Rates gesetzten vertraglichen Grenzen - über konzeptionelle Initiativen den Barcelona-Prozess weiter vorantrieb. Das Europäische Parlament hat auf Grund seiner derzeit noch begrenzten Kompetenzen zumindest die Möglichkeit, als demokratisches Gewissen Missstände aufzuzeigen. ${ }^{24}$ In einem insgesamt extrem

20 Bisher ist nur ein Fall mangelnder horizontaler Kohärenz bekannt geworden, als in der Endphase der Verhandlungen über ein EMAA mit Jordanien die Kommission eine Klausel zur jährlichen Überprüfung der Importquote für Tomatenmark vereinbart hatte, die eine von Spanien angeführte Gruppe von Mitgliedstaaten heftig kritisierte. Sie bestanden - trotz der bereits vollzogenen Paraphierung des EMAA im April 1997 - auf einer Wiederaufnahme der Verhandlungen und der Streichung der jährlichen Überprüfung.

21 Vgl. hierzu ausführlich Ingeborg Tömmel: Das politische System der EU, München 2003.

22 Vgl. Schmid, Les institutions européennes dans le fonctionnement du PEM.

23 Claire Spencer: The EU and Common Strategies: The Revealing Case of the Mediterranean, in: European Foreign Affairs Review 1/2001, S. 31-51; Miguel Ángel Medina Abellán: The Common Strategy on the Mediterranean Region: Evidence and Analysis, Obs-Working Paper, No. 28: Obervatori de Politíca Exterior, Universitat Autónoma de Barcelona 2002.

24 Erwan Lannon: Parlements et societé civile dans la sécurité euro-méditerranéenne, Lissabon: EuroMeSCo Paper Nr. 19, Lissabon 2002. 
komplexen Feld der europäischen Außenpolitik ist es im Laufe der letzten 15 Jahre gelungen, aus einer weit gehend unkoordinierten Politik mit niedrigem - auf Handelsbeziehungen reduziertem - Profil das Programm einer europäischen Mittelmeerpolitik zu entwerfen und umzusetzen.

Vertikale Kohärenz? Konditionierung am Beispiel der europäisch-algerischen Beziehungen

Programmatisch hat die Union ihre neue Mittelmeerpolitik an die Achtung demokratischer Prinzipien und der Menschenrechte in den Partnerstaaten gekoppelt. ${ }^{25}$ In sämtlichen Mittelmeer-Assoziationsabkommen ist festgelegt, dass die Achtung der Menschenrechte und demokratischen Grundwerte Grundlage der inneren wie zwischenstaatlichen Beziehungen sind und die EMAAs bei Verstößen gegen diese Prinzipien suspendiert werden können. Dem müssen bilaterale Konsultationen vorausgehen, die mit dem Ziel einer Einigung geführt werden sollen. Bei Maßnahmen haben diejenigen Vorrang, die das Abkommen am wenigsten verletzen. ${ }^{26}$

Fragen der Konditionierung sind ein Politikfeld, das anfällig für ,Extratouren` von Mitgliedstaaten ist. Auf Grund der hohen politischen Symbolik, die solchen Maßnahmen zugewiesen wird, und deren Rückwirkung auf die bilateralen Beziehungen geht es den EU-Ländern oft darum, hier noch einmal die außenpolitischen Prärogativen eines Nationalstaates zu betonen. Dies lässt sich besonders am Fall der Beziehungen zwischen der Europäischen Union und Algerien beobachten, handelt es sich doch hier um die Frage, ob Frankreich seine Sonderrolle beibehält - und damit die Mittelmeerpolitik vertikale Kohärenz vermissen lässt.

Seit de Gaulle stand die , politique arabe“ als Versuch regionaler Hegemonialpolitik für das Streben nach einer vor allem von den USA unabhängigen Rolle Frankreichs. ${ }^{27}$ Frankreich spielte folglich bis Anfang der 1990er Jahre im Rahmen der EG-Mittelmeerpolitik eine solitäre Rolle. Paris reagierte auf den Putsch des algerischen Militärs im Dezember 1991 und den darauf folgenden blutigen inneren Auseinandersetzungen in der gewohnten Weise: Es unterstützte das neue Regime wie vorher das alte. ${ }^{28}$ Die Reaktionen aus Brüssel zeichneten sich demgegenüber durch Unverbindlichkeit aus. Zwischen 1993 und 1997 veröffentlichte der Rat lediglich drei Erklärungen zur Lage in Algerien, in denen er sich für den politischen Dialog zwischen den verschiedenen politischen Kräften aussprach, die Beachtung der Menschenrechte anmahnte und alle Gewalt verurteilte. Allein das Europäische Parlament forderte zu Beginn des Jahres 1995 den Rat auf, mit einer Gemeinsamen Aktion die Aufnahme des Dialogs zwischen Regierung und Opposition in Algerien zu unterstützen, und plädierte kurz darauf für den sofortigen Stopp der militärischen Kooperation mit Algerien.

In den letzten Jahren der Ära Mitterrand gab es erste Veränderungen in der französischen Algerienpolitik, als Reaktion auf Anschläge und Flugzeugentführungen durch islamistische Extremisten im Winter 1994, die die Erfolglosigkeit der bisherigen Algerienpolitik deutlich

25 Vgl. Richard Gillespie/Laurence Whitehead: European Democracy Promotion in North Africa: Limits and Prospects, in: Democratization 1/2002, S. 192-206.

26 Elena Fierro: Legal Basis and Scope of the Human Rights Clauses in EC Bilateral Agreements: Any Room for Positive Interpretation?, in: European Law Journal 1/2001, S. 41-68.

27 Vgl. zum Folgenden: Laurent Meyrede: France's Foreign Policy in the Mediterranean, in: Stelios Stavridis/ Theodore Couloumbis/Thanos Verimis/Neville Waites (Hrsg.): The Foreign Policies of the European Union's Mediterranean States and Applicant Countries in the 1990s, London 1999, S. 40-72; und Camille BonoraWeismann: France and the Algerian Conflict. Issues in Democracy and Political Stability, 1988-1995, Aldershot 2003.

28 Vgl. insgesamt Melanie Morisse-Schillbach: L'Europe et la question algérienne, Paris 1999; und Jean-François Daguzan: France, Democratization and North Africa, in: Democratization 4/2002, S. 135-148. 
machten. Die französische Regierung versuchte, das Algerienproblem auf die Agenda der Union zu bringen, auch um sich auf europäischer Ebene Unterstützung für ihre Vorstellungen einer neuen Politik des Dialogs und der Mäßigung zu holen. Präsident Chirac forcierte den Wandel; bereits im November 1995 glichen sich die offiziellen Reaktionen auf die ,gelenkte' Präsidentschaftswahl in Algerien zwischen Brüssel und Paris an. ${ }^{29}$ Die französische Regierung lobte einerseits Fortschritte in Richtung Demokratisierung, betonte aber andererseits auch die Notwendigkeit weiterer Fortschritte und die Dringlichkeit eines bilateralen politischen Dialoges. Die Stellungnahme der Union, in der angekündigt wurde, die zukünftige Kooperation von der innenpolitischen Entwicklung des Landes abhängig machen zu wollen, wies in die gleiche Richtung. Diese Angleichung der Positionen und Sichtweisen zeigte sich auch, als der französische Außenminister im Juli 1996 in Algier explizit die Europäische Union sowie die Euro-Mediterrane Partnerschaft als Rahmen für einen politischen Dialog mit Algerien bezeichnete. Die französische Regierung unterstützte durch vertrauliche Kanäle die Reise der EU-Troika nach Algerien im Februar desselben Jahres sowie einer Delegation des Europäischen Parlamentes. Ergebnis dieser Politik war die Institutionalisierung des politischen Dialoges zwischen Algerien und der Union.

Als es im September 1997 in Algerien zu einer neuen Welle von Gewalt kam, veröffentlichte die EU-Ratspräsidentschaft eine Erklärung, in der sie die Massaker verurteilte. Sie betonte zwar, die Union werde die Assoziationsverhandlungen im Rahmen der Euro-Mediterranen Partnerschaft fortsetzen, fügte aber hinzu, dass man erwarte, dass die Regierung die kritische Situation mit, Weisheit und Mäßigung ' behandele, und erinnerte daran, dass der Terrorismus mit Respekt für die Menschenrechte bekämpft werden müsse.

Der Ton zwischen Brüssel und Algier verschärfte sich zusehends, was Ende 1997 eine einseitige Aussetzung der Assoziationsverhandlungen durch die Union zur Folge hatte. Die Kommission begründete dies - neben Differenzen in Sachfragen - mit der durch den Anstieg der Gewalt ausgelösten politischen und menschenrechtlichen Lage. Sie verband dies aber zugleich mit dem Angebot, auf höherer politischer Ebene den Dialog mit Algerien in Gang bringen zu wollen, um danach die Verhandlungen über das Assoziationsabkommen wieder aufnehmen zu können. Es kam zu Besuchen des algerischen Außenministers in Brüssel und der EU-Troika in Algier. Sie bestand darauf, Algerien müsse einen ständigen Dialog über politische Reformen mit der Europäischen Union führen, erst dann könnten die Verhandlungen wieder aufgenommen werden. Nicht durchsetzen konnte sich die Kommission mit der Forderung nach einer UN-Kommission zur Untersuchung der Gewalt in Algerien. Dieser Vorschlag wurde nicht nur von den Betroffenen abgelehnt, sondern auch von Spanien und Italien, die sich auf EU-Ebene zwar nicht klar dagegen ausgesprochen hatten, ihn aber in der UN-Kommission für Menschenrechte abblockten. Die mangelnde vertikale Kohärenz spiegelte die Uneinigkeit unter den Mitgliedstaaten darüber wieder, wer die eigentlichen Urheber der Gewalt in Algerien seien, islamistische Terrorgruppen und/oder von Regierung und Militär gestützte Sicherheitsdienste.

Die Kommission erarbeitete einen Kompromiss zwischen den unterschiedlichen Positionen. Man einigte sich auf einen Verzicht auf eine öffentliche Bloßstellung (, shaming') der algerischen Regierung bei gleichzeitiger Aufforderung, sie müsse für eine Entspannung der innenpolitischen Situation sorgen. Diese neue Politik wurde sofort angesichts der klaren Manipulation der algerischen Präsidentschaftswahlen im April 1999 auf die Probe gestellt. Man war zerstritten über eine angemessene Reaktion, wobei die alten Differenzen zwischen denjeni-

29 Victor Manceron: La nouvelle politique arabe de Jacques Chirac ou l'art du paradoxe, in: Relations internationales et stratégiques, Nr.25/1997, 110-120. 
gen EU-Mittelmeerländern, die für Zurückhaltung plädierten, und den anderen Mitgliedstaaten, die eine schärfere Tonart forderten, wieder auftraten. Schließlich einigte man sich auf Bestreben Frankreichs und der Kommission auf eine moderate Reaktion, unter anderem mit dem Argument, die Union müsse - trotz der Wahlfälschungen - die Absicht der Regierung unterstützen, mit der Opposition Gespräche aufzunehmen.

In der Folge intensivierten sich die französisch-algerischen Beziehungen mit regelmäßigen Treffen auf hoher Ebene. So kam es im Frühjahr 2000 zur Wiederaufnahme der Verhandlungen über das Assoziationsabkommen im Rahmen der Mittelmeerpartnerschaft, das im April 2002 unterzeichnet wurde.

Nach einem anfänglich inkohärenten Verhalten, das durch Diskrepanzen zwischen den Erklärungen aus Brüssel und insbesondere aus Paris gekennzeichnet war, agieren nunmehr in der Algerienpolitik Kommission und Rat auf der Grundlage einer Übereinstimmung in den Einschätzungen und Interessen, in die auch Frankreich eingeschlossen ist. Sie lassen sich folgendermaßen umreißen: Vor allem die südlichen EU-Mitgliedstaaten fürchten, eine konsequente Anwendung der Konditionierungsklauseln könnte destabilisierende Wirkungen auf die Region haben, indem islamistisch-fundamentalistische Kräfte von einer Schwächung staatlicher Autorität profitierten. ${ }^{30}$ Vernünftiger sei es, auf den langsamen Weg der Reform von oben zu setzen, auf die langfristigen Wirkungen der Freihandelszone und der politischen wie gesellschaftlichen Dialogpolitik. Einige Mitgliedstaaten, vor allem aus Skandinavien, setzen einen leicht anderen Akzent und plädieren für eine stärkere Konditionierung der Mittelmeerpolitik, vertreten aber diese Position im Rat und gegenüber der Kommission nicht so nachhaltig, dass von einem von der Mehrheitsmeinung abweichendem Verhalten gesprochen werden kann. Die Kommission teilt in der Konditionierungsfrage die Position der südlichen EU-Mittelmeerstaaten. Sie verfolgt in erster Linie den Kurs einer weit gehenden Marktliberalisierung, von der sie sich Spill-over-Effekte in die politischen und gesellschaftlichen Bereiche erhofft. Sie bevorzugt die politisch weniger ambitionierten Kooperationsprojekte aus Angst vor den möglichen destabilisierenden Folgen einer zu starken Unterstützung nicht-staatlich kontrollierter Gruppierungen. ${ }^{31}$ Sie betont die rechtliche Bindung, die sich aus Verträgen für beide Seiten ergäben. Diese Strategie wird gegen die Kritik aus dem Parlament oder von gesellschaftlichen Initiativen mit politischen Deklarationen abgesichert, in denen Forderungen von Menschenrechtsgruppen und des Europäischen Parlamentes nach einer stärkeren Berücksichtigung der Menschenrechte aufgenommen werden.

Gemeinsamer Nenner dieser unterschiedlichen Interessenakzentuierungen ist die EU-Politik der ,positiven Sanktionierung ' im Rahmen einer Politik der ,Partnerschaft' ${ }^{\prime}{ }^{32}$ eine Strategie, die Frankreich mitgestaltet, die seine Beziehungen zu Algerien aber auch in Richtung einer Europäisierung verändert hatte. Dieser Kompromiss wurde maßgeblich von der Kommission vorgedacht und vorbereitet. ${ }^{33}$ Bestrafende Sanktionen sind nur bei extremen Verstößen gegen die Achtung der Menschenrechte vorgesehen, ansonsten gelten die Vertragseinhaltung und die Thematisierung demokratischer und menschenrechtlicher Defizite im Rahmen

30 Vgl. Eberhard Kienle: Destabilization Through Partnership? Euro-Mediterranean Relations After the Barcelona Declaration, in: Mediterranean Politics 2/1998, 1-20; und Bechir Chourou: The Challenge of Democracy in North Africa, in: Democratization 4/2002, S. 135-148.

31 Annette Jünemann: From the Bottom to the Top: Civil Society and Transnational NGOs in the Euro-Mediterranean Partnership, in: Democratization 1/2002, S. 87-105.

32 Dorothée Schmid: Interlinkages Within the Euro-Mediterranean Partnership. Linking Economic, Institutional and Political Reform: Conditionality Within the Euro-Mediterrranean Partnership, EuroMeSCo Paper 27, Lissabon 2003.

33 Judith Kelley: New Wine in Old Wineskins: Policy Adaption in the European Neighborhood Policy, erscheint 2006 im Journal of Common Market Studies. 
,politischer Dialoge' auf allen Ebenen, von der gesellschaftlichen bis zur höchsten gouvernementalen. Diejenigen Staaten jedoch, die Fortschritte bei der Menschenrechtsrealisierung und bei ,Good Governance ' vorweisen können, werden mit zusätzlicher Hilfe belohnt, so dass sie relativ mehr Mittel erhalten als diejenigen, die weiter Menschenrechte und Demokratie missachten.

\section{Euro-Mediterrane Partnerschaft und Europäische Nachbarschaftspolitik}

Seit kurzem ist der Barcelona-Prozess Teil der Europäischen Nachbarschaftspolitik (ENP). Auch bei dieser Entwicklung wiederholen sich Muster, wie sie schon bei der Konzeption der europäischen Mittelmeerpolitik beobachtet werden konnten. Zunächst machten sich einige (nördliche) Mitgliedstaaten sowie die Kommission Gedanken über die Konsequenzen der Osterweiterung für angrenzende Staaten wie die Ukraine, Weißrussland und Moldawien. ${ }^{34}$ Ziel sollte sein, diese Staaten näher an die Europäische Union heranzuführen, ohne ihnen jedoch eine Beitrittsperspektive zu geben. Dies rief sofort die südlichen Mitgliedstaaten auf den Plan, die die Einbeziehung der Mittelmeerländer in die ENP forderten. Dieses Ansinnen traf sich mit der Einschätzung der Kommission, dass der Barcelona-Prozess, der wieder einmal zu lahmen drohte, dringend eines neues Impulses bedürfe. ${ }^{35}$ Mit dem Irak-Krieg, dem weiter ungelösten Konflikt zwischen Israel und den Palästinensern, dem Ausscheiden Zyperns und Maltas sowie der Türkei aus der EMP durch die EU-Mitgliedschaft beziehungsweise die anvisierten Beitrittsverhandlungen war eine Neuorientierung notwendig geworden.

Bei der Formulierung der ENP füllte die Kommission wieder ihre konzeptionelle Vorreiterrolle aus, die sie schon im Vorfeld der Barcelona-Konferenz eingenommen hatte. Sie knüpfte an zwei Erfahrungen an, die sie in die neue ENP einbrachte. Zum einen versuchte sie, die Methoden und Erfahrungen, die bei den Verhandlungen mit den mittel- und osteuropäischen Staaten mit der Konditionierung gemacht worden waren, auch für Situationen nutzbar zu machen, in denen die Beitrittsperspektive kein Anreiz für Partnerstaaten ist, Politik, Gesellschaft und Wirtschaft zu reformieren. Im Ergebnis wurde in den ENP-Dokumenten die bereits in der EMP erprobte Strategie der positiven Sanktionierung sehr viel deutlicher als Mittel der Förderung von Menschenrechten und Demokratie hervorgehoben. Zum anderen adaptierte sie für die ENP die Korb-Struktur und inhaltliche Ausfüllung der Mittelmeerpartnerschaft und bot - damit noch über das Ziel der Freihandelszone hinausgehend denjenigen Staaten, die dies wollen und können, die Einbeziehung in den Europäischen Binnenmarkt an.

\section{Die Europäische Union - Ein kollektiver Akteur in der Euro-Mediterranen Partnerschaft}

Durch die Analyse des nunmehr zehnjährigen Barcelona-Prozesses wird erkennbar, dass eine entscheidende Voraussetzung für die EU-Akteursqualität die Involvierung der Europäischen Kommission in die - pfeilerübergreifende - Außenpolitikformulierung und -implementierung ist. Mit ihrer ,Verfahrensmacht' gestaltet die Kommission Außenpolitik. Ihre konzeptionellen Vorgaben waren die Bedingung für ein zunehmend koordiniertes Handeln im Rahmen einer gemeinsamen Zielvorstellung und Interessendefinition der Mitgliedstaa-

34 Vgl. Michael Emerson: European Neighbourhood Policy: Strategy or Placebo?, CEPS Working Document, No. 215, Centre for European Policy Studies, November 2004 Brüssel.

35 Vgl. Michael Emerson/Gergana Noutcheva: From Barcelona Process to Neighbourhood Policy. Assesments and Open Issues, CEPS Working Document, Nr. 220, Centre for European Policy Studies, Brüssel 2005; und Raffaella A. Del Sarto/Tobias Schumacher: From EMP to ENP: What's at Stake with the European Neighbourhood Policy towards the Southern Mediterranean, in: European Foreign Affairs Review 1/2005, S. 17-38. 
ten, anfängliche horizontale und vertikale Kohärenzdefizite wurden immer mehr zu Gunsten einer kollektiven Akteursqualität abgelöst. Zwar ziehen die EU-Institutionen nicht immer vollständig an einem Strang, sie handeln aber auch nicht offen gegeneinander. Es zeigte sich, dass in den Fällen, in denen die Kommission eine hervorgehobene Rolle spielt, also beispielsweise bei den Assoziationsverhandlungen, die Kohärenz größer und damit die Akteursqualität eindeutiger ist. Demgegenüber ist diese weniger ausgeprägt, wenn es um Politikfelder geht, die vorwiegend im Kompetenzbereich der Mitgliedstaaten liegen und für diese einen hohen politischen Stellenwert haben. Die stärkere Involvierung der Kommission scheint somit eine Voraussetzung für eine kohärente Außenpolitik zu sein, sie wirkt sich in den Bereichen besonders aus, in denen es weniger um operative Fragen von ,high politics“ als um langfristige Konzeptionen und Strategien - und um das Tagesgeschäft der Umsetzung - geht.

Der Aufsatz ist im Rahmen des Projektes ,Die Gemeinsame Außen- und Sicherheitspolitik (GASP) der Europäischen Union - Intergouvernementales Netzwerk oder kollektiver Akteur? ' entstanden, das am Institut für Europäische Politik in Berlin und an der Hessischen Stiftung Friedens- und Konfliktforschung in Frankfurt/Main durchgeführt wurde.

Das gesamte Nomos Programm $~$ suchen $"$ finden $\rightsquigarrow$ bestellen unter www.nomos.de

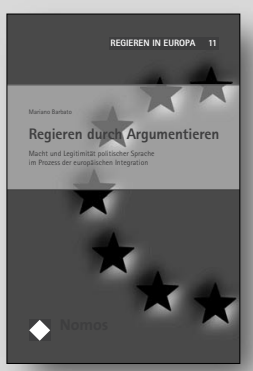

\section{Regieren durch Argumentieren}

Macht und Legitimität politischer Sprache im Prozess der europäischen Integration

Von Dr. Mariano Barbato, Universität Bamberg

2005, 244 S., brosch., 48,-€, ISBN 3-8329-1392-O

(Regieren in Europa, Bd. 11)

In Europa zählen Argumente, so der Autor. Doch was meint Argumentieren und wie lauten die europäischen Argumente? Barbato entwickelt eine neue Methode zur Narration der politischen Argumentationszusammenhänge und erschließt hierdurch Zugänge zu den zentralen europäischen Argumenten. Der Autor legt somit eine philosophisch wohl durchdachte innovative Methode vor. 